\title{
AVALIAÇÃO GEOQUÍMICA DE BIOMARCADORES OCLUÍDOS EM ESTRUTURAS ASFALTÊNICAS
}

\author{
Débora de Almeida Azevedo*, Tais Freitas da Silva e Daniel Bastos da Silva \\ Departamento de Química Orgânica, Instituto de Química, Universidade Federal do Rio de Janeiro, Av. Athos da Silveira Ramos, \\ 149, 21941-909 Rio de Janeiro - RJ, Brasil
}

Recebido em 7/8/08; aceito em 9/4/09; publicado na web em 26/8/09

\begin{abstract}
GEOCHEMICAL EVALUATION OF OCCLUDED BIOMARKERS IN ASPHALTENIC STRUCTURES. Asphaltenes from two Brazilian crude oils were submitted to mild oxidation to disrupt their structure, releasing the occluded oil. The released hydrocarbons were compared with those from the original crude oil, and used to evaluate the alteration of the oils, especially as a result of biodegradation, but also thermal maturity. The crude oils used are depleted in $n$-alkanes, which are usually related to biodegradation. However, the released products from the corresponding asphaltenes have $n$-alkane distributions from $\mathrm{nC}_{10}$ to $\mathrm{nC}_{40}$, suggesting a protection effect from biodegradation. The $\mathrm{m} / z, 191$ mass chromatograms showed higher relative intensities for tricyclic terpanes than the hopanes in the crude in comparison with the released ones.
\end{abstract}

Keywords: asphaltenes; biodegradation; occluded biomarkers.

\section{INTRODUÇÃO}

A caracterização adequada dos constituintes do petróleo é uma informação indispensável para a determinação do comportamento termodinâmico e é, portanto, de grande importância para todas as operações de produção, desde a estimativa das reservas existentes até os projetos para o seu transporte, refino e a distribuição de seus produtos. A natureza química, a quantidade e a interação destas frações com os outros constituintes do petróleo influenciam significativamente algumas propriedades e fenômenos importantes como, por exemplo, a precipitação de parafinas em petróleos sujeitos a ambientes frios (linhas de transporte de petróleo em águas profundas) e a desestabilização e deposição de asfaltenos no reservatório, na coluna de produção e em linhas de transporte, devido a alterações nas composições e condições termodinâmicas de temperatura e pressão. A formação de sólidos durante a produção de petróleo traz prejuízos que variam do aumento dos gastos energéticos, devido ao aumento de viscosidade, até mesmo a interrupção da produção.

Entre os constituintes na fração pesada de petróleo que vem despertando interesse e atenção, estão os asfaltenos.

O termo asfalteno é utilizado para designar macromoléculas do petróleo, de alta massa molecular. ${ }^{1-4}$ Dada a sua complexidade, os asfaltenos são definidos em relação a sua solubilidade, sendo insolúveis em $n$-alcanos de baixa massa molecular, como hexano, pentano ou heptano e solúveis em tolueno ou benzeno. ${ }^{5,6}$ Em relação ao seu aspecto, são sólidos amorfos com coloração marrom escura.

Em geral, é conhecido que a estrutura molecular básica dos asfaltenos é formada por unidades poliaromáticas condensadas juntamente com cadeias alifáticas nos ou entre os anéis aromáticos. Devido a suas características estruturais, os asfaltenos podem se aglomerar formando espécies de "gaiolas", podendo, assim, ocluir outros compostos presentes nos óleos. Esses compostos, uma vez ocluídos, ficam protegidos pela estrutura asfaltênica de alterações secundárias como, por exemplo, biodegradação e maturação, possivelmente por limitar seu contato com micro-organismos e agentes catalisadores presentes nos reservatórios. Por isso, acredita-se que sejam remanescentes do óleo original. A liberação dessas espécies ocluídas vem demonstrando ser uma nova ferramenta para o estudo geoquímico de óleos intensamente biodegradados.

\footnotetext{
*e-mail: debora@iq.ufrj.br
}

Estudos recentes têm utilizado oxidação branda para a liberação de biomarcadores de asfaltenos..$^{7-9}$ Durante o tratamento oxidativo, qualquer grupo alifático que esteja diretamente ligado à molécula de asfalteno quando for craqueado, estará "marcado", por exemplo, como ácido carboxilíco alifático. A oxidação branda despolimeriza a molécula, separa os grupamentos aromáticos, e as pontes que ligam este grupamento são convertidas em cadeias periféricas com grupos carboxílicos terminais. Portanto, os hidrocarbonetos obtidos após a oxidação branda não são aqueles que estavam quimicamente ligados à molécula de asfalteno, mas são remanescentes do óleo original ocluídos em sua estrutura.

Após o petróleo ser acumulado no reservatório, podem ocorrer mudanças na sua composição. As alterações que ocorrem nos óleos crus alteram seu valor econômico e algumas vezes mascaram os estudos de correlação óleo-óleo e óleo-rocha geradora. As modificações nos parâmetros moleculares, devido a essas alterações, dificultam a identificação de fonte e maturidade em óleos degradados.

Óleos maturos e parcialmente biodegradados assemelham-se a óleos imaturos na sua composição, dificultando a distinção destes em uma análise por cromatografia gasosa.

A alteração térmica ocorre quando o óleo depositado sofre ação do aumento de temperatura. Este fenômeno associado ao tempo de residência favorece a formação de hidrocarbonetos leves devido ao craqueamento dos componentes mais pesados. ${ }^{10}$

A biodegradação é a alteração seletiva de determinados tipos de hidrocarbonetos por bactérias presentes no reservatório. Ocorre, normalmente, em reservatórios localizados em áreas onde houve contato com águas meteóricas. Essas águas meteóricas carregam certos microorganismos aos reservatórios juntamente com oxigênio e nutrientes.

A biodegradação altera a matéria orgânica original e existem vários trabalhos na literatura que discutem os efeitos da biodegradação em óleos. ${ }^{11-15}$ A biodegradação, por micro-organismos aeróbicos e/ ou anaeróbicos, ocorre inicialmente pela perda de compostos mais leves ( $n$-alcanos de baixa massa molecular) seguida de compostos de maior massa molecular.

Os parâmetros de correlações geoquímicas são baseados nos perfis obtidos para várias classes de compostos, através de análises por cromatografia gasosa acoplada à espectrometria de massas. A diversidade de estruturas dos biomarcadores permite supor que sedimentos orgânicos diferentes possam apresentar composição 
variável para pelo menos alguns desses grupos de estruturas. Esta pressuposição é empregada em correlação entre diferentes amostras de óleos de uma mesma bacia, ou comparando-se a constituição de óleos com a matéria orgânica de rochas geradoras potenciais.

Vários parâmetros são utilizados para avaliar o nível de maturação em amostras de óleos. Grande parte destes parâmetros está baseada nas reações de isomerização de biomarcadores. A isomerização na posição C-22 (22R e 22S) nos homo-hopanos (hopanos com 31 a 35 átomos de carbono; H31-H35) pode ser utilizada para avaliar o grau de evolução térmica de óleos. É calculada a proporção entre o epímero de configuração biológica (22R) e geológica (22S) pela razão $22 \mathrm{~S} /(22 \mathrm{R}+22 \mathrm{~S})$. Os valores variam de 0-0,6 (faixa de equilíbrio de 0,57-0,62) durante a evolução térmica, alcançando este valor antes do pico de geração de óleo. ${ }^{10,16}$

Os parâmetros $20 \mathrm{~S} /(\mathrm{S}+\mathrm{R}) \mathrm{C} 29$ esterano e $\beta \beta /(\beta \beta+\alpha \alpha) \mathrm{C} 29$ esterano também são indicativos de maturidade térmica. ${ }^{17,18} \mathrm{~A}$ comparação entre estes dois indicadores pode ser utilizada para frações geradas a diferentes temperaturas e tempos de uma ou mais rochas geradoras. Com o aumento da maturação térmica, a isomerização no C-20 de configuração $5 \alpha, 14 \alpha, 17 \alpha(\mathrm{H})-\mathrm{C} 29$ esterano, existente nos precursores esteroidais dos organismos vivos, é convertida em uma mistura de configurações $\mathrm{R}$ e $\mathrm{S}$. A razão de isomerização aumenta de zero para aproximadamente 0,5 , sendo o valor de equilíbrio de $0,52-0,55 .{ }^{10,19}$ A biodegradação em óleos interfere nos valores desta razão, devido à remoção seletiva de epímeros. Com o aumento da maturação, a isomerização nas posições C-14 e C-17 do C29 esterano 20S e 20R aumenta a razão $\beta \beta /(\beta \beta+\alpha \alpha)$ de zero para aproximadamente 0,7 , cujos valores de equilíbrio se encontram entre 0,67 e $0,71 . .^{10,16}$

$\mathrm{Na}$ biodegradação, os $n$-alcanos são preferencialmente degradados em relação aos outros compostos, tais como os isoprenoides acíclicos, pristano e fitano. As razões entre os isoprenoides, pristano e fitano, e os $n$-alcanos (pristano/ $n \mathrm{C} 17$ e fitano/ $n \mathrm{C} 18$ ) são utilizadas para avaliar o grau de biodegradação. Em óleos de biodegradação moderada, essas razões apresentam valores mais elevados que os óleos não-biodegradados. Porém, em óleos que apresentam biodegradação severa, as razões tendem a zero devido à ausência destes compostos, observando apenas uma curva na linha base do cromatograma mais conhecida como mistura complexa de compostos não-resolvidos (Unresolved Complex Mixture -UCM). ${ }^{10}$

O produto da degradação dos hopanos são os hopanos desmetilados, cuja série (chamada de 25-nor-hopanos) pode ser observada no cromatograma de massas do íon $\mathrm{m} / z$. 177. A presença desses compostos, assim como elevada abundância relativa de $n$-alcanos e isoprenoides em óleos, tem sido utilizada como um indicador de mistura de óleos intensamente biodegradados com óleos não-biodegradados provenientes possivelmente de um segundo pulso de geração. ${ }^{10,13}$

A presença do composto 25-nor-hopano está relacionada com a maioria dos óleos severamente biodegradados, pois é resultado da remoção da metila 25 do carbono 10 do hopano por ação das bactérias. ${ }^{10,14}$ Entretanto, alguns trabalhos mencionam a presença do 25-nor-hopano em extratos de rocha geradora, sugerindo que este composto também seja gerado durante a diagênese, e sua concentração em óleos aumenta durante o processo de biodegradação..$^{14,20}$

Portanto, este trabalho teve como objetivo estudar as características geoquímicas de duas amostras de óleos da Bacia de Campos (denominadas de $\mathrm{CP}$ e CL) através da precipitação dos asfaltenos dos óleos utilizando-se $n$-heptano e posterior abertura da macroestrutura dos asfaltenos obtidos, por reação de oxidação com $\mathrm{NaIO}_{4}-\mathrm{NaHPO}_{4}$, com vista a liberar os biomarcadores ocluídos em seu interior. A caracterização geoquímica dos hidrocarbonetos saturados (biomarcadores) liberados de dentro da macroestrutura asfaltênica foi feita através de análises como cromatografia gasosa por detecção por ionização em chama (CG-DIC) e por cromatografia gasosa acoplada à espectrometria de massas (CG-EM).

\section{PARTE EXPERIMENTAL}

\section{Materiais e solventes}

Toda a vidraria foi lavada com água e sabão e posteriormente submersa e deixada em solução de Extran alcalino 2\% (Merck, Rio de Janeiro, Brasil) por $24 \mathrm{~h}$, para remoção de qualquer contaminante orgânico. Os solventes utilizados, tais como diclorometano, metanol, n-hexano e tolueno, foram de grau cromatográficos e obtidos da TediaBrazil (Rio de Janeiro, Brasil). A sílica gel foi adquirida da Merck (Rio de Janeiro, Brasil). Esta foi previamente extraída com diclorometano em aparelhagem tipo Soxhlet por 48 h, para remoção de qualquer contaminante orgânico.

\section{Precipitação dos asfaltenos}

As amostras de óleo cru (500 mg) foram pesadas e $20 \mathrm{~mL}$ de $n$-heptano foram adicionados ao óleo. Agitou-se gentilmente o sistema por $30 \mathrm{~s}$ obtendo-se asfaltenos disperso no malteno. A mistura foi deixada em repouso por $24 \mathrm{~h}$. Após, o sistema foi centrifugado durante 5 min sob rotação de $2000 \mathrm{rpm}$ em uma centrífuga Excelsa Baby II Modelo 206-R e, em seguida, o asfalteno foi separado por decantação. ${ }^{21,22} \mathrm{O}$ asfalteno obtido foi lavado quatro vezes nas mesmas condições descritas anteriormente para eliminar possíveis interferentes que podem ficar adsorvidos em sua superfície.

\section{Análise elementar}

Os asfaltenos foram submetidos à análise elementar, em triplicata, em um aparelho Fleshea 1112 Series, com detector TCD, usando hélio como gás de arraste numa vazão de $130 \mathrm{~mL} / \mathrm{min}$ e $\mathrm{O}_{2}$ como gás de combustão numa vazão de $100 \mathrm{~mL} / \mathrm{min}$.

\section{Degradação dos asfaltenos por reação oxidativa}

Toda a massa de asfalteno precipitada foi transferida para um balão de $100 \mathrm{~mL}$ e completamente solubilizada com $50 \mathrm{~mL}$ de tolueno e $2 \mathrm{~mL}$ de diclorometano. Os reagentes $\mathrm{NaIO}_{4}(0,1 \mathrm{~g})$ e $\mathrm{NaH}_{2} \mathrm{PO}_{4}$ $(0,1 \mathrm{~g})$ foram previamente dissolvidos em $5 \mathrm{~mL}$ de água destilada $\mathrm{e}$ adicionados ao balão contendo o asfalteno. O sistema permaneceu sob agitação magnética à temperatura ambiente por $12 \mathrm{~h} .^{7,23}$

\section{Reprecipitação}

Após a reação de oxidação, as fases orgânica e aquosa foram separadas em funil de separação de $250 \mathrm{~mL}$. Descartou-se a fase aquosa. A fase orgânica sofreu três extrações com $10 \mathrm{~mL}$ de água (para a completa retirada do sal), seca com $\mathrm{Na}_{2} \mathrm{SO}_{4}$ (anidro) e filtrada. A remoção do solvente foi realizada por evaporação quase a secura em rotaevaporador a temperatura de $40{ }^{\circ} \mathrm{C}$.

Adicionou-se $20 \mathrm{~mL}$ de $n$-heptano ao produto obtido da oxidação dos asfaltenos (acima descrito), seguido de centrifugação (nas mesmas condições da precipitação de asfaltenos) e decantação. Este procedimento foi repetido três vezes. O solvente foi evaporado quase a secura (aproximadamente $100 \mu \mathrm{L}$ ) em rotaevaporador a temperatura de $40{ }^{\circ} \mathrm{C}$ e a fração residual, contendo os compostos solúveis em $n$ heptano, foi fracionada por cromatografia líquida.

\section{Cromatografia líquida}

Os óleos crus (aproximadamente $100 \mathrm{mg}$ ) e os óleos ocluídos (produtos da liberação a partir da oxidação dos asfaltenos diretamente do procedimento anterior) foram fracionados em coluna de sílica gel 
60 (2,5 g de sílica; $0,063-0,200 \mathrm{~mm}$; Merck) ativada a $120^{\circ} \mathrm{C}$ durante $12 \mathrm{~h}$. As frações foram eluidas com $10 \mathrm{~mL}$ de $n$-hexano para os hidrocarbonetos saturados, $10 \mathrm{~mL}$ de $n$-hexano/diclorometano (8:2) para os hidrocarbonetos aromáticos e $10 \mathrm{~mL}$ de diclorometano/metanol (9:1) para os compostos polares (NSO). Estas foram recolhidas em balões de $25 \mathrm{~mL}$, seguido de evaporação em rotaevaporador.

Todo o método (todas as etapas acima descritas) também foi realizado em uma amostra branco (sem a presença de uma amostra de óleo mas com todos os solventes e reagentes) para a avaliação de possível contaminação. Não foi observado qualquer contaminante através das análises cromatográficas.

\section{Cromatografia gasosa por detecção por ionização em chama (CG-DIC)}

As amostras de óleo total, assim como as frações dos hidrocarbonetos saturados (tanto do obtido por fracionamento do óleo quanto do ocluso obtido por reação oxidativa da fração asfaltênica) foram analisadas por cromatografia gasosa por detecção por ionização em chama (CG-DIC). O equipamento utilizado foi Agilent Technologies 6890 , com uma coluna capilar de sílica fundida recoberta com DB-5 (J \& W; $30 \mathrm{~m}$ x 0,25 mm d.i.; espessura de fase de 0,25 $\mu \mathrm{m}$ ). A programação de temperatura do forno foi de $40{ }^{\circ} \mathrm{C}(1 \mathrm{~min})$ a 310 ${ }^{\circ} \mathrm{C}, 6{ }^{\circ} \mathrm{C} /$ min e mantido em isoterma a $310^{\circ} \mathrm{C}$ por $15 \mathrm{~min}$. Temperatura do injetor foi de $290{ }^{\circ} \mathrm{C}$ e do detector de $330^{\circ} \mathrm{C}$. Foi utilizado hidrogênio como gás de arraste e injeção com divisão de fluxo de 1/10. Foi injetado $1 \mu \mathrm{L}$ da amostra diluída a um volume final de 1 $\mathrm{mL} \operatorname{com} n$-hexano.

\section{Cromatografia gasosa acoplada à espectrometria de massas (CG-EM)}

As frações dos hidrocarbonetos saturados foram analisadas por cromatografia gasosa acoplada à espectrometria de massas (CG-EM) em um instrumento Agilent Technologies 5973 interfaceado ao cromatógrafo Agilent Technologies 6890 utilizando uma coluna capilar de sílica fundida recoberta com DB-5 (J\&W; $30 \mathrm{~m} \mathrm{X} \mathrm{0,25} \mathrm{mm} \mathrm{d.i.;}$ espessura de fase de $0,25 \mu \mathrm{m})$. A programação de temperatura foi: temperatura inicial de $70{ }^{\circ} \mathrm{C}$; programação de temperatura de $70-170$ ${ }^{\circ} \mathrm{C}$ a $20^{\circ} \mathrm{C} / \mathrm{min} ; 170-300{ }^{\circ} \mathrm{C}$ a $2^{\circ} \mathrm{C} /$ min e mantido em isoterma à 300 ${ }^{\circ} \mathrm{C}$ por $10 \mathrm{~min}$, temperatura do injetor a $290{ }^{\circ} \mathrm{C}$ e da interface a $300{ }^{\circ} \mathrm{C}$. Foi utilizado hélio como gás carreador e injeção sem divisão de fluxo por $1 \mathrm{~min}$, ionização por impacto de elétrons a $70 \mathrm{eV}$. Utilizou-se o monitoramento seletivo de íons (MSI; SIM = Single Ion Monitoring) como modo de análise para os íons $m / z$ 85, 191, 177, 217 e 218, assim como análise em varredura linear (Scan) na faixa de 50-550 Daltons. A identificação dos compostos foi realizada com base em dados da literatura, perfil cromatográfico de amostras de referência, tempo de retenção relativo e espectro de massas.

\section{RESULTADOS E DISCUSSÃO}

\section{Análise elementar e tratamento oxidativo}

A quantidade de asfalteno obtido, por precipitação, e sua composição elementar (CHN) estão apresentadas na Tabela 1. A análise elementar mostrou que o teor de carbono e hidrogênio é similar para as duas amostras, com uma porcentagem de carbono de aproximadamente $85 \%$ e uma razão de aproximadamente 1:10 de H:C para os asfaltenos das duas amostras de óleos. Observou-se que o óleo CP possui um teor mais elevado de asfaltenos em relação ao óleo CL, 41,8 e 34,7\%, respectivamente. Os teores mais elevados de carbono em relação ao hidrogênio podem ser explicados em virtude da alta aromaticidade das estruturas asfaltênicas. A composição dos óleos ocluídos pelos asfaltenos está apresentada na Tabela 2, enquanto a percentagem de hidrocarbonetos saturados (Sat), aromáticos (Arom) e compostos polares (Pol) para o óleo CP foram de 42,7; 19,8; 37,6\% e para o óleo CL de 53,$6 ; 23,7$ e $22,7 \%$, respectivamente. A fração ocluída pelo asfalteno da amostra CL apresentou um teor mais elevado de hidrocarbonetos saturados $(21,2 \%)$ quando comparado ao asfalteno do óleo CP $(15,2 \%)$. Os percentuais destas frações em relação à massa inicial de asfaltenos precipitados, para a amostra $\mathrm{CP}$, foram de 3,0\% de hidrocarbonetos saturados, $6,5 \%$ de hidrocarbonetos aromáticos e $10,3 \%$ de resinas, enquanto que para a amostra CL foram de 3,0\% de hidrocarbonetos saturados, $2,0 \%$ de hidrocarbonetos aromáticos e 9,2\% de resinas. Os diferentes valores podem ser atribuídos às diferenças estruturais entre os asfaltenos dessas amostras, ou ainda que o asfalteno do óleo CL seja mais reativo face ao tratamento oxidativo.

Tabela 1. Quantidade (\% em massa) e composição elementar (\%) dos asfaltenos obtidos por precipitação

\begin{tabular}{lcccc}
\hline $\begin{array}{l}\text { Amostras } \\
\text { de óleos }\end{array}$ & $\begin{array}{l}\text { Média (n=3) } \\
\text { \% em massa }\end{array}$ & $\mathrm{N}$ & $\mathrm{C}$ & $\mathrm{H}$ \\
\hline CP & 41,78 & nd & 85,40 & 8,69 \\
CL & 34,70 & nd & 84,54 & 8,88 \\
\hline
\end{tabular}

Tabela 2. Composição (\%) do óleo liberado por oxidação dos asfaltenos (SAT, ARO e NSO) e seus respectivos rendimentos relativos $(\%)$ à massa inicial de asfaltenos $\left(\mathrm{SAT}_{\mathrm{R}}, \mathrm{ARO}_{\mathrm{R}}\right.$ e $\left.\mathrm{NSO}_{\mathrm{R}}\right)$

\begin{tabular}{lcccccc}
\hline Amostras & SAT & ARO & NSO & SAT $_{\mathrm{R}}$ & $\mathrm{ARO}_{\mathrm{R}}$ & $\mathrm{NSO}_{\mathrm{R}}$ \\
\hline $\mathrm{CP}$ & 15,2 & 32,9 & 51,9 & 3,0 & 6,5 & 10,3 \\
$\mathrm{CL}$ & 21,2 & 14,1 & 64,7 & 3,0 & 2,0 & 9,2 \\
\hline
\end{tabular}

\section{Análise de óleo total (whole oil)}

A Figura 1 apresenta os perfis cromatográficos dos hidrocarbonetos das amostras de óleo CP (A e B) e CL (C e D). As figuras A e C são correspondentes aos hidrocarbonetos livres e B e D aos hidrocarbonetos ocluídos pelos respectivos asfaltenos.

Pela análise dos cromatogramas observa-se que o perfil cromatográfico da amostra CL é de um óleo relativamente parafínico (Figura 1C). As parafinas são observadas na faixa de $n \mathrm{C} 10$ a $n \mathrm{C} 40$ com predominância de compostos de baixa massa molecular, sendo que a maior abundância é observada para $n \mathrm{C} 14$.

Para o óleo CP foi observada a quase total ausência de parafinas (Figura 1A), com elevação da linha de base devido à predominância de compostos de maior massa molecular que não foram resolvidos cromatograficamente. A ausência de $n$-alcanos é interpretada como indícios de remoção por micro-organismo, isto é, de biodegradação.

Nos cromatogramas obtidos pela análise dos hidrocarbonetos ocluídos pelos respectivos asfaltenos, observa-se uma maior abundância de $n$-alcanos, com predominância dos de baixa massa molecular para o óleo CP (Figura 1B) e uma distribuição bimodal para o óleo CL (Figura 1D), evidenciando a preservação de $n$-alcanos de baixa e alta massa molecular pela estrutura asfaltênica em relação à biodegradação. A análise dos cromatogramas de óleo total nos fornece uma visão rápida e eficiente do fenômeno de proteção dos compostos ocluídos pela estrutura asfaltênica.

A Tabela 3 mostra os valores dos parâmetros pristano/fitano $(\mathrm{Pr} / \mathrm{Fi})$, pristano $/ n \mathrm{C} 17(\mathrm{Pr} / n \mathrm{C} 17)$ e fitano $/ n \mathrm{C} 18(\mathrm{Fi} / n \mathrm{C} 18)$ obtidos pela análise dos cromatogramas dos óleos livres e oclusos das amostras CP e CL, os quais foram calculados através da relação entre as áreas dos compostos em questão. Os menores valores observados para as razões Pr/ $n \mathrm{C} 17$ e Fi/ $n \mathrm{C} 18$ nas frações ocluídas pelos asfaltenos são devidos à preservação dos 
compostos $n \mathrm{C} 17$ e $n \mathrm{C} 18$ pela estrutura asfaltênica. Se compararmos os valores destes parâmetros com os valores do óleo livre, verifica-se que há uma tendência a conclusões diferentes com os diferentes resultados.
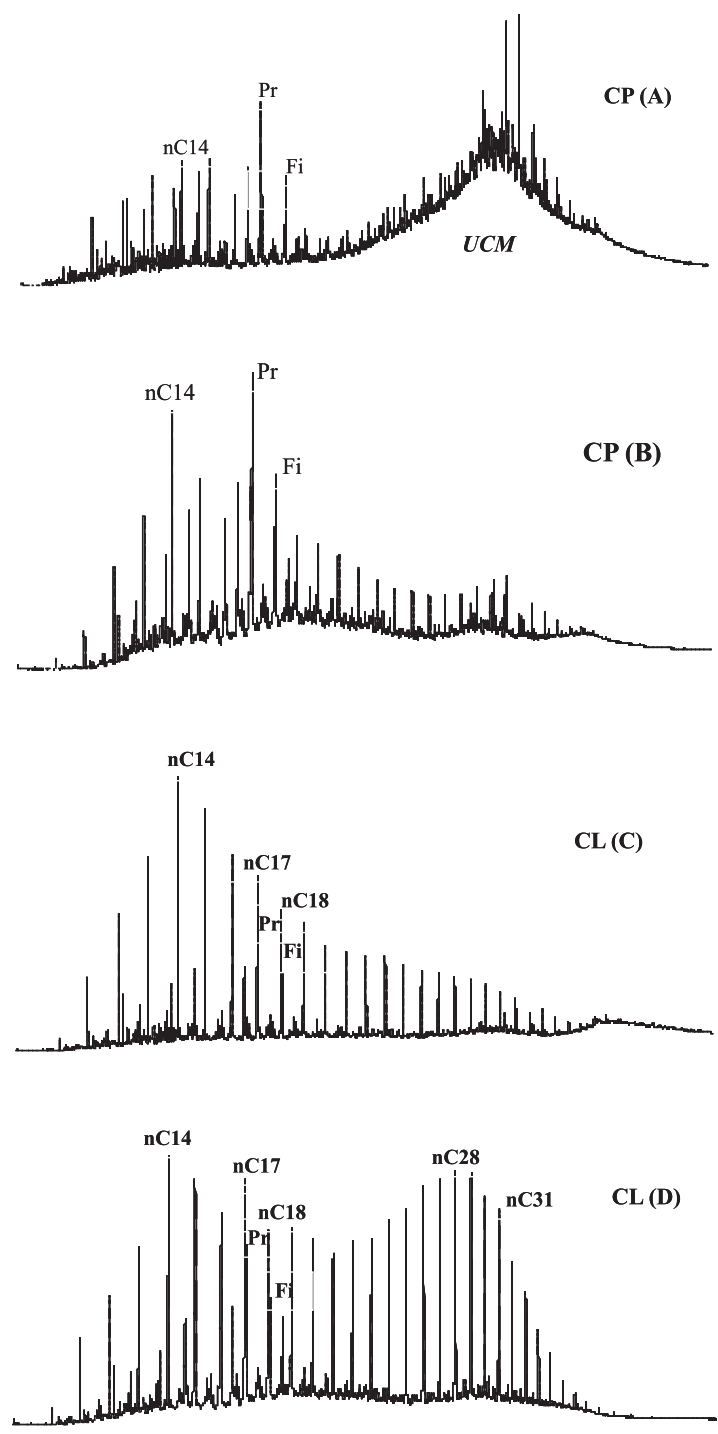

Figura 1. Cromatogramas dos hidrocarbonetos saturados das amostras de óleo CP (A e B) e CL (C e D): (A e C) hidrocarbonetos livres; (B e D) hidrocarbonetos ocluídos pelos respectivos asfaltenos

\section{Cromatografia gasosa acoplada à espectrometria de massas (CG-EM)}

A distribuição dos biomarcadores relativos aos terpanos e esteranos, determinada por CG-EM nas frações de hidrocarbonetos saturados dos óleos livres e dos produtos liberados após a oxidação dos asfaltenos (oclusos) nas amostras CP e CL, encontra-se na Figura 2. As razões geoquímicas foram determinadas com base nas áreas dos compostos em questão.

Os cromatogramas de massas do íon $\mathrm{m} / \mathrm{z}$ 191, característico dos terpanos, para as amostras CP e CL estão apresentados na Figura 2. Pela análise dos cromatogramas observa-se a presença dos terpanos tricíclicos, tetracíclicos e pentacíclicos, tanto nas frações de hidrocarbonetos saturados dos óleos livres, quanto nas frações ocluídas pelos respectivos asfaltenos.

Ao comparar os perfis cromatográficos, observa-se uma diferença na abundância relativa entre os terpanos tricíclicos e pentacíclicos, sendo
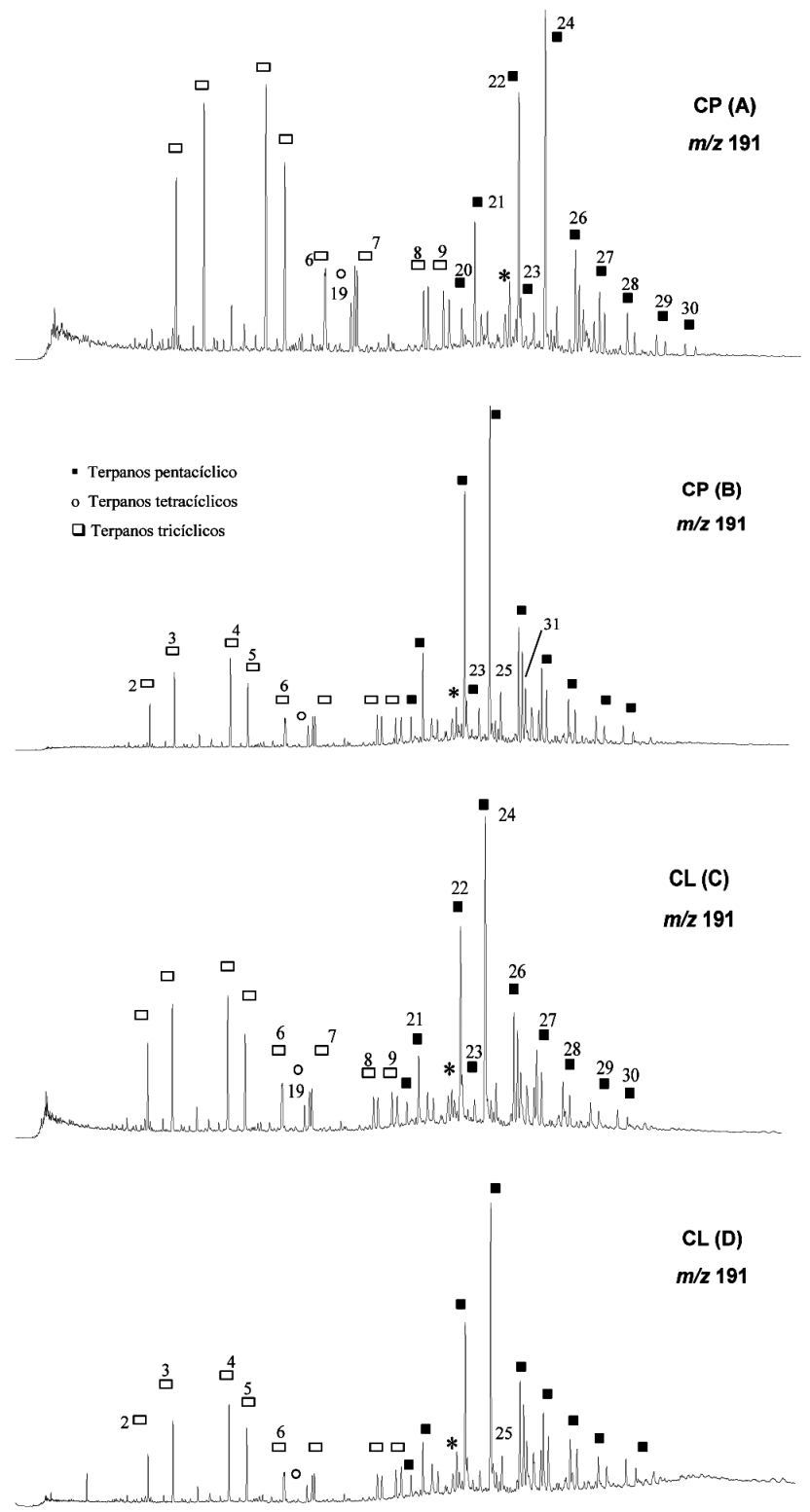

Figura 2. Cromatogramas de massas do íon $\mathrm{m} / \mathrm{z}$ 191, característicos de terpanos, das amostras de óleos $C P(A$ e B) e CL (C e D): A e C) terpanos "livres" no óleo; B e D) terpanos ocluídos nos asfaltenos. Obs: Os números correspondem os compostos da Tabela 4

estes últimos menos intensos nas frações de hidrocarbonetos saturados dos óleos livres. Isto pode ser explicado pelo fato desses serem mais suscetíveis à biodegradação do que os terpanos tricíciclos. Porém, uma vez ocluídos pela estrutura asfaltênica, ficam protegidos dessa biodegradação. Isto pode ser melhor visualizado pela análise da razão terpano tricíclico C23 / 17 $\alpha, 21 \beta$-hopano (Tr23/H30) para o óleo CP, onde para os hidrocarbonetos livres no óleo apresenta uma razão de 2,0 (Tabela 3), enquanto esta razão para os hidrocarbonetos ocluídos pelo respectivo asfalteno sofre um decréscimo para 0,6 por preservar o $\mathrm{H} 30$.

Em trabalho prévio, ${ }^{7}$ no cromatograma dos terpanos da amostra de óleo (livres) da Bacia de Songliao (China) foi observada a ausência dos terpanos tricíclicos de C20 até C26 e a presença dos mesmos nos biomarcadores ocluídos, enquanto que em $\mathrm{CP}$ os mesmos terpanos estão presentes nos biomarcadores livres e ocluídos.

A relação entre os terpanos tricíclicos e pentacíclicos também sofre influência do processo de maturação, ${ }^{24}$ onde óleos mais evoluídos 
Tabela 3. Valores dos parâmetros de biomarcadores dos óleos livres (L) e ocluídos (O) dos óleos CP e CL

\begin{tabular}{|c|c|c|c|c|}
\hline Parâmetros & $\mathrm{HS}_{\mathrm{CP}}(\mathrm{L})$ & $\mathrm{HS}_{\mathrm{CP}}(\mathrm{O})$ & $\mathrm{HS}_{\mathrm{CL}}(\mathrm{L})$ & $\mathrm{HS}_{\mathrm{CL}}(\mathrm{O})$ \\
\hline $\mathrm{Ts} /(\mathrm{Ts}+\mathrm{Tm})$ & 0,23 & 0,22 & 0,26 & 0,29 \\
\hline $\mathrm{C} 29 \mathrm{Ts} /(\mathrm{H} 29+\mathrm{C} 29 \mathrm{Ts})$ & 0,23 & 0,23 & 0,10 & 0,10 \\
\hline Ts/H30 & 0,10 & 0,07 & 0,07 & 0,07 \\
\hline $\operatorname{Tr} 23 / \mathrm{H} 30$ & 2,00 & 0,60 & 0,32 & 0,27 \\
\hline $\mathrm{H} 33 \mathrm{~S} /(\mathrm{S}+\mathrm{R})$ & 0,67 & 0,55 & 0,55 & 0,53 \\
\hline $\mathrm{S} /(\mathrm{S}+\mathrm{R}) \alpha \alpha \alpha \mathrm{C} 29$ & 0,38 & 0,18 & 0,46 & 0,47 \\
\hline$\beta \beta /(\beta \beta+\alpha \alpha)(\mathrm{S}+\mathrm{R}) \mathrm{C} 29$ & 0,43 & 0,45 & 0,49 & 0,53 \\
\hline $\begin{array}{l}\text { Dia } 27(\mathrm{~S}+\mathrm{R}) / \alpha \alpha \alpha \mathrm{C} 27 \\
(\mathrm{~S}+\mathrm{R})\end{array}$ & 0,65 & 0,50 & 0,47 & 0,44 \\
\hline $\mathrm{Pr} / n \mathrm{C} 17$ & 4,42 & 1,75 & 0,78 & 0,61 \\
\hline $\mathrm{Fi} / n \mathrm{C} 18$ & 2,49 & 2,17 & 0,83 & 0,69 \\
\hline $\mathrm{Pr} / \mathrm{Fi}$ & 1,83 & 1,49 & 1,43 & 1,26 \\
\hline
\end{tabular}

HS = Hidrocarbonetos saturados. Ts/(Ts+Tm) = razão entre as áreas dos compostos $18 \alpha(\mathrm{H})-22,29,30$-Tris-nor-neo-hopano sobre $(18 \alpha(\mathrm{H})$ 22,29,30-Tris-nor-neo-hopano $+17 \alpha(\mathrm{H})$-22,29,30-Tris-nor-hopano); C29Ts/(H29 + C29 Ts) = razão das áreas do 18 $\alpha(\mathrm{H})-30$-nor-neo-hopano C29 sobre $(17 \alpha, 21 \beta(\mathrm{H})-30$-nor-hopano $+18 \alpha(\mathrm{H})$-30-nor-neo-hopano C29); Ts/H30 = razão das áreas do composto 18 $\alpha(\mathrm{H})$-22,29,30-Tris-norneo-hopano sobre $17 \alpha, 21 \beta(\mathrm{H})$-hopano; Tr23/H30 = razão Terpano Tricíclico C23 sobre 17 $\alpha, 21 \beta(\mathrm{H})$-hopano; H33S/(S+R) = 17 $\alpha, 21 \beta(\mathrm{H})-29$ tris-homo-hopano $(22 \mathrm{~S}) /(17 \alpha, 21 \beta(\mathrm{H})-29$-tris-homo-hopano $(22 \mathrm{~S})+17 \alpha, 21 \beta(\mathrm{H})$-29-tris-homo-hopano $(22 \mathrm{R}))$; $\mathrm{S} / \mathrm{S}+\mathrm{R} \alpha \alpha \alpha \mathrm{C} 29=5 \alpha, 14 \alpha, 17 \alpha-$ estigmastano 20S / $(5 \alpha, 14 \alpha, 17 \alpha$-estigmastano $20 \mathrm{~S}+5 \alpha, 14 \alpha, 17 \alpha$-estigmastano $20 \mathrm{R}) ; \beta \beta /(\beta \beta+\alpha \alpha)(\mathrm{S}+\mathrm{R}) \mathrm{C} 29=(5 \alpha, 14 \beta, 17 \beta$-estigmastano $20 \mathrm{R}+5 \alpha, 14 \beta, 17 \beta$-estigmastano $20 \mathrm{~S}) /([5 \alpha, 14 \beta, 17 \beta$-estigmastano $20 \mathrm{R}+5 \alpha, 14 \beta, 17 \beta$-estigmastano $20 \mathrm{~S})]+[5 \alpha, 14 \alpha, 17 \alpha$-estigmastano $20 \mathrm{R}+5 \alpha, 14 \alpha, 17 \alpha$-estigmastano 20S]); Dia 27(S+R)/ $\alpha \alpha \alpha \mathrm{C} 27(\mathrm{~S}+\mathrm{R})=$ razão $(13 \beta, 17 \alpha$-diacolestano 20S+13 $\beta, 17 \alpha$-diacolestano 20R) / $(5 \alpha, 14 \alpha, 17 \alpha$-colestano $20 \mathrm{R}+5 \alpha, 14 \alpha, 17 \alpha$-colestano $20 \mathrm{~S}) ; \mathrm{Pr} / n \mathrm{C} 17=$ razão Pristano $/ n$-alcano C17; Fi/nC18 = razão Fitano / n-alcano C18; $\mathrm{Pr} / \mathrm{Fi}=$ razão Pristano $/$ Fitano

termicamente apresentam maior abundância dos terpanos tricíclicos, o que também pode estar colaborando para os resultados observados em nossas amostras, ou seja, compostos pentacíclicos uma vez ocluídos pela estrutura asfaltênica ficam protegidos tanto dos processos de biodegradação quanto dos efeitos térmicos.

Outro parâmetro bastante utilizado para avaliar o grau de maturidade térmica de óleos é a razão Ts/(Ts+Tm) [18 $\alpha(\mathrm{H})-22,29,30-T r i s-n o r-n e o-$ hopano/(18 $\alpha(\mathrm{H})-22,29,30$-Tris-nor-neo-hopano $+17 \alpha(\mathrm{H})-22,29,30-$ Tris-nor-hopano)], pois o composto Ts é mais estável termicamente. Porém, esta razão também é influenciada pelo ambiente de deposição da rocha geradora. ${ }^{10,25}$ Não foram observadas diferenças significativas na relação desses compostos entre as frações de hidrocarbonetos livres nos óleos e aqueles ocluídos pelos respectivos asfaltenos (Tabela 3). A biodegradação dos compostos Ts e Tm geralmente ocorre na mesma velocidade, permanecendo sempre a razão inicial. ${ }^{10}$

A razão Ts/H30 também é utilizada na avaliação de maturidade térmica (Tabela 3). Segundo Volkman e colaboradores, ${ }^{26}$ esta razão tende a aumentar com o aumento da maturidade térmica. Porém, assim como foi observado para a razão $\mathrm{Ts} /(\mathrm{Ts}+\mathrm{Tm})$ não houve diferença significativa entre os valores observados para a fração de hidrocarbonetos livres no óleo e ocluídos pelos respectivos asfaltenos.

Muitos autores sugerem que a abundância dos compostos relativos ao17 $\alpha$-Hopano seja usada para maturidade térmica. ${ }^{27,28}$ A relação entre H29 (17 $\alpha, 21 \beta(\mathrm{H})-30$-nor-hopano) e C29Ts (18 $\alpha(\mathrm{H})$-30-norneo-hopano C29), assim como a razão entre Ts e Tm, também é utilizada na avaliação de evolução térmica de óleos, uma vez que o C29Ts é mais estável termicamente do que o composto H29 (Figura 2; picos 23 e 22, respectivamente). Com isso, a razão C29Ts/(H29+ C29Ts) aumenta com o aumento da maturidade térmica. Os valores observados, tanto para a amostra CP quanto CL, são similares entre as frações de hidrocarbonetos livres no óleo e ocluídos pelos asfaltenos, indicando mesmo grau de evolução térmica.
A distribuição de diasteranos e esteranos regulares pode ser observada pela análise do cromatograma de massas do íon $\mathrm{m} / \mathrm{z}, 217$ (Figura 1S - Material Suplementar).

A razão entre os isômeros do composto C29 esterano ( $\alpha \alpha \alpha$ 20S/ (20S+20R), picos 11 e 14; e $\beta \beta /(\alpha \alpha+\beta \beta)$, picos 12 e 13 da Figura $1 S)$ é um parâmetro bastante usual na avaliação de maturidade térmica dos óleos. ${ }^{10}$ Assim como foi observado na análise dos terpanos, não houve diferenças significativas entre valores observados nas frações de hidrocarbonetos livres e ocluídos. Os parâmetros usuais de evolução térmica não demonstraram mudanças significativas para os óleos livres e ocluídos, levando a concluir que não houve mudança em relação à evolução térmica.

A biodegradação parcial dos esteranos do óleo pode resultar no crescimento da razão $\alpha \alpha \alpha 20 \mathrm{~S} /(20 \mathrm{~S}+20 \mathrm{R})$ acima de 0,55 por causa da remoção seletiva do epímero $\alpha \alpha \alpha$ 20R pelas bactérias. ${ }^{10}$ Pela análise dessa razão, nossos resultados indicam que os esteranos não foram afetados pelo processo de biodegradação. Porém, na análise da razão $\beta \beta /(\alpha \alpha+\beta \beta)$ para o esterano C29 (Tabela 3) observa-se que no óleo $\mathrm{CP}$ houve um decréscimo dessa razão, de 0,38 na fração dos hidrocarbonetos livres no óleo para um valor de 0,18 na fração ocluída pelo respectivo asfalteno. Isso pode ser devido à preservação do epímero biológico de configuração $\alpha \alpha \alpha$ pela estrutura asfaltênica, o qual é mais suscetível à biodegradação do que seu isômero geológico $\alpha \beta \beta$.

Pela análise do cromatograma de massas do íon $\mathrm{m} / z, 177$ (Figura 2S - Material Suplementar), observa-se a presença de 25,28,30-trisnor-hopano nas frações de biomarcadores livres. Este pode ser produto de biodegradação do 28,30-bis-nor-hopano, ${ }^{29}$ porém ele também pode ser encontrado em óleos não biodegradados. Neste caso, a presença deste composto, tanto na fração de biomarcadores livres quanto ocluídos, tem sido interpretada como indicativo que este é formado durante o processo diagenético e uma alta concen- 
Tabela 4. Identificação dos biomarcadores e abreviações, referentes ao íon $\mathrm{m} / z, 191$

\begin{tabular}{|c|c|c|c|c|}
\hline Número & Nomes & Abreviação & Fórmula Molecular & Massa Molecular \\
\hline 1 & Terpano Tricíclico C19 & $\operatorname{Tr} 19$ & С19H34 & 262 \\
\hline 2 & Terpano Tricíclico C20 & $\operatorname{Tr} 20$ & $\mathrm{C} 20 \mathrm{H} 36$ & 276 \\
\hline 3 & Terpano Tricíclico C21 & $\operatorname{Tr} 21$ & C21H38 & 290 \\
\hline 4 & Terpano Tricíclico C23 & $\operatorname{Tr} 23$ & $\mathrm{C} 23 \mathrm{H} 42$ & 318 \\
\hline 5 & Terpano Tricíclico C24 & $\operatorname{Tr} 24$ & $\mathrm{C} 24 \mathrm{H} 44$ & 332 \\
\hline 6 & Terpano Tricíclico C25 (S/R) & $\operatorname{Tr} 25(\alpha$ e $\beta)$ & $\mathrm{C} 25 \mathrm{H} 46$ & 346 \\
\hline 7 & Terpano Tricíclico C26 (S/R) & $\operatorname{Tr} 26(\alpha$ e $\beta)$ & $\mathrm{C} 26 \mathrm{H} 48$ & 360 \\
\hline 8 & Terpano Tricíclico C28 (S/R) & $\operatorname{Tr} 28(\alpha$ e $\beta)$ & $\mathrm{C} 28 \mathrm{H} 52$ & 388 \\
\hline 9 & Terpano Tricíclico C29 (S/R) & $\operatorname{Tr} 29(\alpha$ e $\beta)$ & C29H54 & 402 \\
\hline 10 & Terpano Tricíclico C30 (S/R) & $\operatorname{Tr} 30(\alpha$ e $\beta)$ & $\mathrm{C} 30 \mathrm{H} 56$ & 416 \\
\hline 11 & Terpano Tricíclico C31 (S/R) & $\operatorname{Tr} 31(\alpha$ e $\beta)$ & C31H58 & 430 \\
\hline 12 & Terpano Tricíclico C33 (S/R) & $\operatorname{Tr} 33(\alpha$ e $\beta)$ & $\mathrm{C} 33 \mathrm{H} 60$ & 444 \\
\hline 13 & Terpano Tricíclico C34 (S/R) & $\operatorname{Tr} 34(\alpha$ e $\beta)$ & C34H62 & 458 \\
\hline 14 & Terpano Tricíclico C35 (S/R) & $\operatorname{Tr} 35(\alpha$ e $\beta)$ & C35H64 & 472 \\
\hline 15 & Terpano Tricíclico C36 (S/R) & $\operatorname{Tr} 36(\alpha$ e $\beta)$ & C36H66 & 486 \\
\hline 16 & Terpano Tricíclico C38 (S/R) & $\operatorname{Tr} 38(\alpha$ e $\beta)$ & C37H68 & 500 \\
\hline 17 & Terpano Tricíclico C39 (S/R) & $\operatorname{Tr} 39(\alpha$ e $\beta)$ & $\mathrm{C} 38 \mathrm{H} 70$ & 514 \\
\hline 18 & Terpano Tricíclico C40 (S/R) & $\operatorname{Tr} 40(\alpha$ e $\beta)$ & $\mathrm{C} 39 \mathrm{H} 72$ & 528 \\
\hline 19 & Terpano Tetracíclico C24 & Tet24 & $\mathrm{C} 24 \mathrm{H} 42$ & 330 \\
\hline 20 & $18 \alpha(H)-22,29,30$-Tris-nor-neo-hopano & Ts & $\mathrm{C} 27 \mathrm{H} 46$ & 370 \\
\hline 21 & $17 \alpha(\mathrm{H})-22,29,30$-Tris-nor-hopano & $\mathrm{Tm}$ & $\mathrm{C} 27 \mathrm{H} 46$ & 370 \\
\hline 22 & $17 \alpha, 21 \beta(\mathrm{H})-30$-nor-hopano & $\mathrm{H} 29$ & $\mathrm{C} 29 \mathrm{H} 50$ & 398 \\
\hline 23 & $18 \alpha(\mathrm{H})-30$-nor-neo-hopano C29 & C29Ts & $\mathrm{C} 29 \mathrm{H} 50$ & 398 \\
\hline 24 & $17 \alpha, 21 \beta(\mathrm{H})$-hopano & $\mathrm{H} 30$ & C30H52 & 412 \\
\hline 25 & $17 \beta, 21 \alpha(\mathrm{H})$-hopano (Moretano) & M30 & $\mathrm{C} 30 \mathrm{H} 52$ & 412 \\
\hline 26 & $17 \alpha, 21 \beta(H)$-29-homo-hopano (22S-22R) & H31 & C31H54 & 426 \\
\hline 27 & $17 \alpha, 21 \beta(\mathrm{H})-29$-bis-homo-hopano (22S-22R) & H32 & $\mathrm{C} 32 \mathrm{H} 56$ & 440 \\
\hline 28 & $17 \alpha, 21 \beta(\mathrm{H})-29$-tris-homo-hopano (22S-22R) & H33 & C33H58 & 454 \\
\hline 29 & $17 \alpha, 21 \beta(\mathrm{H})-29$-tetrakis-homo-hopano (22S-22R) & $\mathrm{H} 34$ & C34H60 & 468 \\
\hline 30 & $17 \alpha, 21 \beta(\mathrm{H})-29$-pentakis-homo-hopano (22S-22R) & $\mathrm{H} 35$ & C35H62 & 482 \\
\hline 31 & Gamacerano & Gam & C30H52 & 412 \\
\hline
\end{tabular}

tração deste composto está relacionada ao ambiente de deposição. ${ }^{10}$ Em estudo prévio, ${ }^{30}$ a presença de $25,28,30-\mathrm{TNH}$ foi detectada em extratos de rochas geradoras da margem continental brasileira, a qual foi relacionada à deposição em ambiente anóxico. Acredita-se que nas duas amostras em estudo, $\mathrm{CP}$ e CL, os compostos desmetilados detectados estejam relacionados ao ambiente de deposição e não à biodegradação ocorrida após a formação do óleo.

\section{CONCLUSÃO}

As amostras de óleos, denominadas de CP e CL, apresentaram alta percentagem relativa de asfaltenos. A avaliação do perfil de hidrocarbonetos parafínicos e biomarcadores demonstrou que os óleos "livres" possuem perfis diferenciados em relação à dos ocluídos. Enquanto que óleo "livre" da amostra CL apresentou predominância das parafinas de baixa massa molecular ( $n$ C12-nC19), o óleo ocluído apresentou máximos de intensidades nas parafinas entre $n \mathrm{C} 12-n \mathrm{C} 19$ e $n \mathrm{C} 24-n \mathrm{C} 31$. Já para $\mathrm{CP}$, o aumento das intensidades relativas dos hidrocarbonetos ocorre em toda a série homóloga dos hidrocarbonetos. Portanto, isso significa que essas parafinas foram protegidas pelas estruturas dos asfaltenos.

Quanto aos parâmetros de maturação e biodegradação relativos aos terpanos e esteranos, pode-se inferir que os dados obtidos indicam que apesar de apresentarem, em sua maioria, valores superiores para os hidrocarbonetos livres, os valores não variaram significantemente entre biomarcadores "livres" e ocluídos. A razão Tr23/H30 apresentou altos níveis nas amostras $\mathrm{CP}$, pois os terpanos tricíclicos são altamente resistentes à biodegradação, e pela avaliação do perfil cromatográfico dos hidrocarbonetos parafínicos desta amostra observou-se que CP é um óleo com elevado nível de biodegradação.

Por fim, os resultados indicam que a ordem de proteção dos hidrocarbonetos ao ataque dos micro-organismos externos para as amostras em estudo foi: hidrocarbonetos parafínicos $>$ terpanos $>$ esteranos. 
Conclui-se por fim que houve proteção dos óleos ocluídos pelas estruturas asfaltênicas, permitindo assim uma análise geoquímica do óleo original.

\section{MATERIAL SUPLEMENTAR}

As Figuras 1S a 3S e Tabela 1S estão disponíveis gratuitamente em http://quimicanova.sbq.org.br, na forma de arquivo PDF.

\section{AGRADECIMENTOS}

Às bolsas recebidas do CNPq, Capes e ANP/PRH-01.

\section{REFERÊNCIAS}

1. Speight, J. G. Em Asphaltes and Asphaltenes; Yen, T. F.; Chilingarian, G. V., eds.; Elsevier: Amsterdam, 1994, cap. 2.

2. Mansoori, G. A.; J. Pet. Sci. Eng. 1997, 17, 101.

3. Calemma, V.; Rausa, R.; D’Antona, P.; Montanari, L.; Energy Fuels 1998, 12, 422.

4. Gawrys, K. L.; Kilpatrick, P. K.; J. Colloid Interface Sci. 2005, 288, 325.

5. Seidl, P. R.; Chrisman, E. C. A. N.; Carvalho, C. C. V.; Leal, K. Z.; Menezes, S. M. C. D.; J. Dispersion Sci. Technol. 2004, 25, 349.

6. Badre, S.; Gonçalves, C. C.; Norinaga, K.; Gustavson, G.; Mullins, O. C.; Fuel 2006, 85, 1.

7. Liao, Z.; Geng, A.; Org. Geochem. 2002, 33, 1477.

8. Liao, Z.; Geng, A.; Graciaa, A.; Creux, P.; Chrostowska, A.; Zhang, Y.; Org. Geochem. 2006, 37, 291.

9. Liao, Z.; Graciaa, A.; Geng, A.; Chrostowska, A.; Creux, P.; Appl. Geochem. 2006, 21, 833.

10. Peters, K. E.; Walters, C. C.; Moldowan, J. M.; The Biomarker Guide: Biomarkers and Isotopes in the Petroleum Exploration and Earth History, $2^{\text {nd }}$ ed., University Press: Cambridge, 2005, vol. 2.

11. Aarssen, B. G. K.; Bastow, T. P.; Alexander, R.; Kagi, R. I.; Org. Geochem. 1999, 30, 1213.
12. Matyasik, I.; Steezko, A.; Philp, R. P.; Org. Geochem. 2000, 31, 1509.

13. Alberdi, M.; Moldowan, J. M.; Peters, K. E.; Dahl, J. E.; Org. Geochem. 2001, 32, 181.

14. Bost, F. D.; Frontera-Suau, R.; McDonald, T. J.; Org. Geochem. 2001, 32, 105.

15. Behar, F.; Penteado, H. L. B.; Lorant, F.; Budzinski, H.; Org. Geochem. 2006, 37, 1042.

16. Jesuino L. S.; Dissertação de Mestrado, Universidade Federal do Rio de Janeiro, Brasil, 2005.

17. Grimalt, J. O.; Campos, P. G.; Berdie, L.; Lopez-Quintero, J. O.; Navarrete-Reyes, L. E. ; Appl. Geochem. 2002, 17, 1.

18. Justwan, H.; Dahl, B.; Isaksen, G. H.; Mar. Pet. Geol. 2006, 23, 213.

19. Seifert, W. K.; Moldowan, J. M. Em Methods in Geochemistry and Geophysics; Jonhs, R.B., ed.; Elsevier: Amsterdam, 1986, vol. 24.

20. Blanc, P.; Connan, J.; Org. Geochem. 1992, 18, 813.

21. Gürgey K.; Org. Geochem. 1998, 29, 1139.

22. Gürgey, K.; Mar. Petrol. Geol. 2003, 20, 1119.

23. Silva, T. F.; Azevedo, D. A; Rangel, M. D.; Fontes, R. A.; Aquino Neto, F. R.; Org. Geochem. 2008, 39, 1249.

24. Seifert, W. K.; Moldowan, J. M.; Geochim. Cosmochim. Acta 1978, 42 , 77.

25. Moldowan, J. M.; Sundararaman, P.; Schoell, M.; Org. Geochem. 1986, 10,915 .

26. Volkman, J. K.; Alexander, R.; Kagi, R. I.; Woodhouse, C. W.; Geochim. Cosmochim. Acta 1983, 47, 785 .

27. Hughes, W. B.; Holba, A. G.; Mueller, D. E.; Richardson, J. S. Em Geochemistry in Exploration of the Norwegian Shelf; Thomas, B. M., ed.; Graham and Trotman: London, 1985.

28. Sofer, Z.; Zumberge, J. E.; Lay, V.; Org. Geochem. 1986, 10, 377; Sofer, Z.; Org. Geochem. 1988, 12, 421.

29. Peters, K. E.; Moldowan, J. M.; Org. Geochem. 1991, 17, 47.

30. Mello, M. R.; Maxwell, J. R. Em Lacustrine basin exploration: case studies and modern analogs; Katz, B. J., ed.; American Association of Petroleum Geologists: Tulsa, 1990. 


\section{AVALIAÇÃO GEOQUÍMICA DE BIOMARCADORES OCLUÍDOS EM ESTRUTURAS ASFALTÊNICAS}

Débora de Almeida Azevedo*, Tais Freitas da Silva e Daniel Bastos da Silva

Departamento de Química Orgânica, Instituto de Química, Universidade Federal do Rio de Janeiro, Av. Athos da Silveira Ramos, 149, 21941-909 Rio de Janeiro - RJ, Brasil
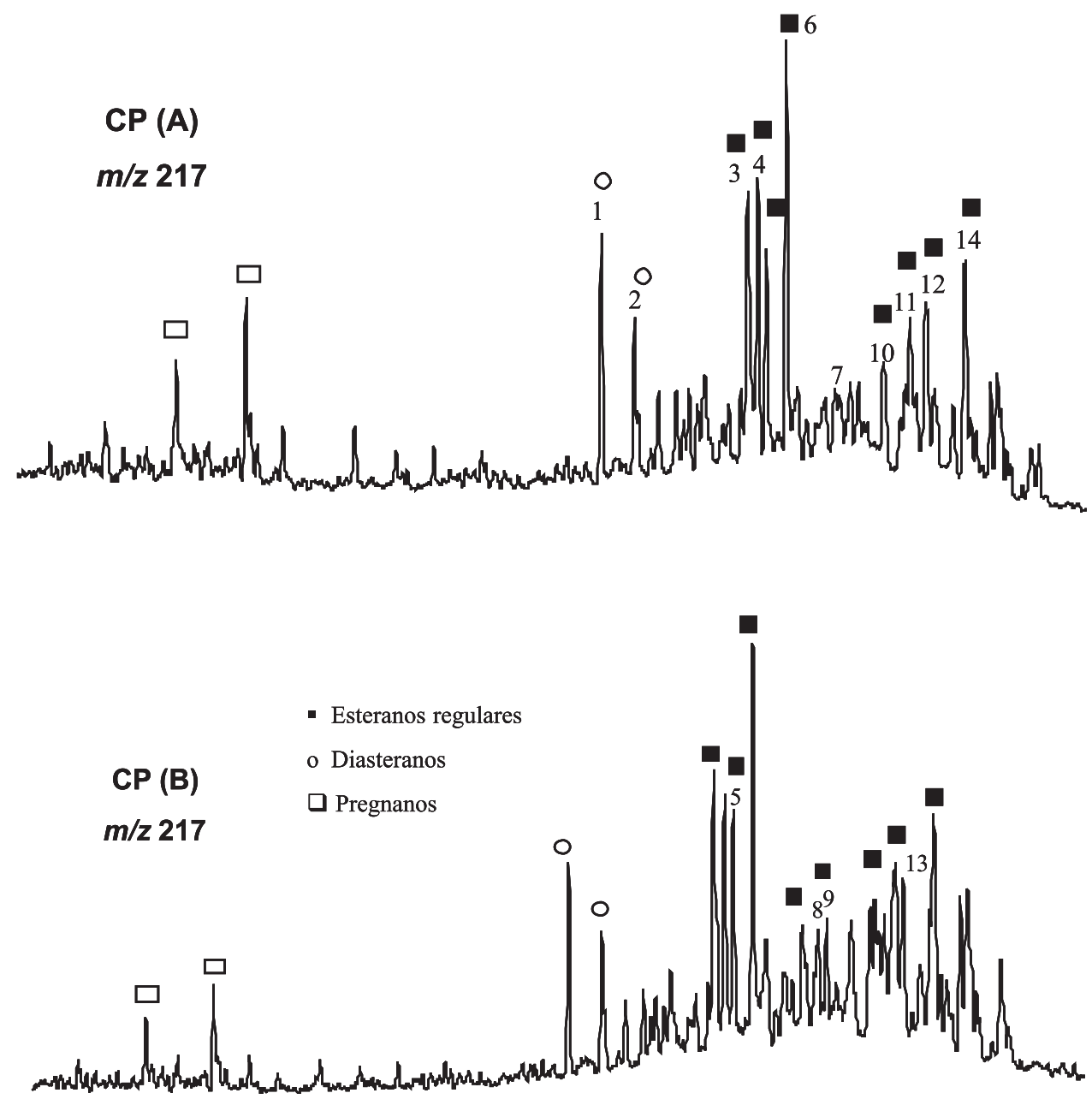

Figura 1S. Cromatogramas de massas do íon $\mathrm{m} / z$ 217, característico de esteranos, da amostra de óleo CP: A) Esteranos livres no óleo, B) Esteranos ocluídos no asfalteno. Obs: Os números correspondem aos compostos da Tabela $1 \mathrm{~S}$

*e-mail: debora@iq.ufrj.br 

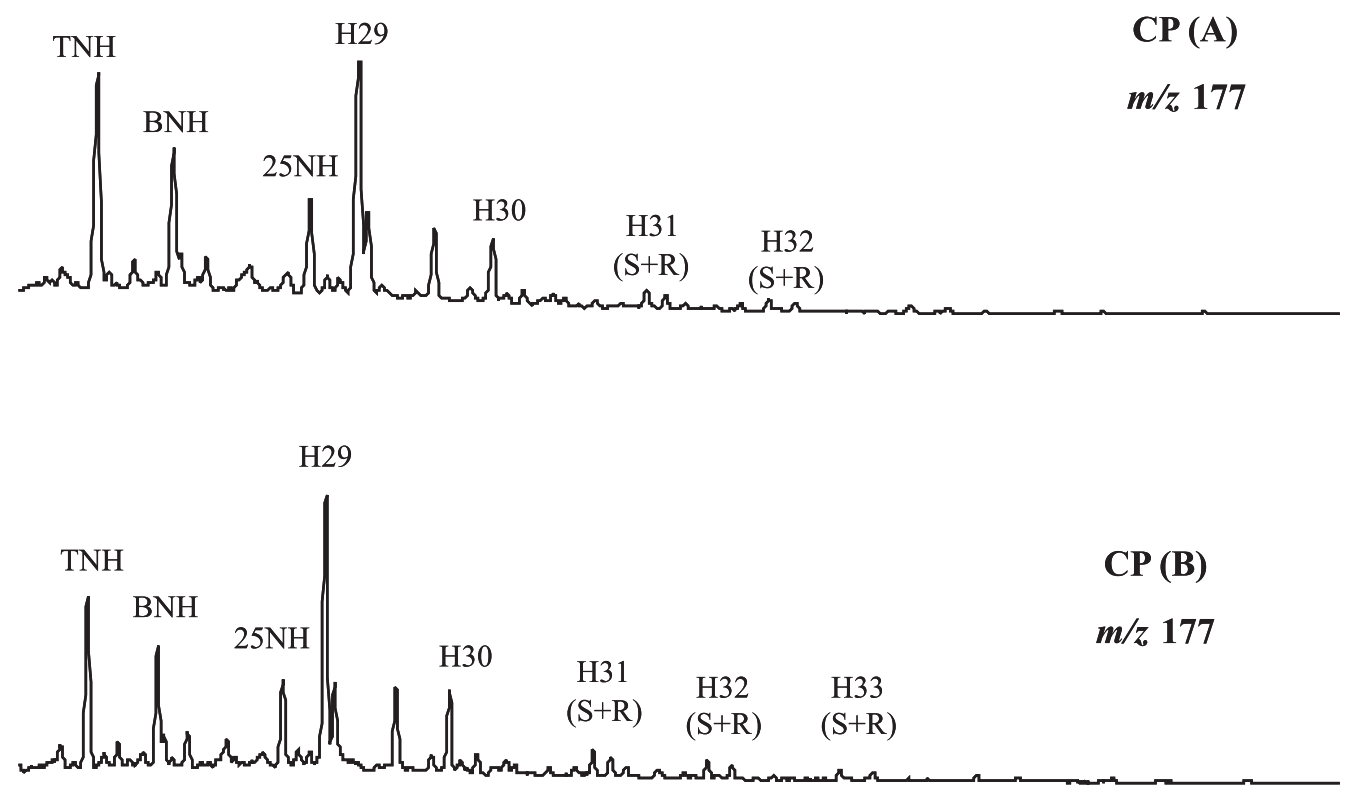

Figura 2S. Cromatogramas de massas do íon m/z 177 característico dos 25-nor-hopanos da amostra de óleo CP: (A) biomarcadores livres e (B) biomarcadores ocluídos. TNH = 25,28,30-Tris-nor-hopano, BNH = 25,30-bis-nor-hopano, 25NH = 25-nor-hopano, H29 = 30-Nor-hopano; H30 = Hopano

Tabela 1S. Identificações dos biomarcadores e abreviações, referentes ao íon $\mathrm{m} / z 217$

\begin{tabular}{|c|c|c|c|c|}
\hline Número & Nomes & Abreviação & Fórmula Molecular & Massa Molecular \\
\hline 1 & $13 \beta, 17 \alpha$-diacolestano $20 \mathrm{~S}$ & Dia $S$ & $\mathrm{C} 27 \mathrm{H} 48$ & 372 \\
\hline 2 & $13 \beta, 17 \alpha$-diacolestano $20 \mathrm{R}$ & Dia R & $\mathrm{C} 27 \mathrm{H} 48$ & 372 \\
\hline 3 & $5 \alpha, 14 \alpha, 17 \alpha$-colestano $20 \mathrm{~S}$ & $\alpha \alpha \alpha S$ & $\mathrm{C} 27 \mathrm{H} 48$ & 372 \\
\hline 4 & $5 \alpha, 14 \beta, 17 \beta$-colestano 20R & $\alpha \beta \beta R$ & $\mathrm{C} 27 \mathrm{H} 48$ & 372 \\
\hline 5 & $5 \alpha, 14 \beta, 17 \beta$-colestano $20 \mathrm{~S}$ & $\alpha \beta \beta S$ & $\mathrm{C} 27 \mathrm{H} 48$ & 372 \\
\hline 6 & $5 \alpha, 14 \alpha, 17 \alpha$-colestano $20 \mathrm{R}$ & $\alpha \alpha \alpha \mathrm{R}$ & $\mathrm{C} 27 \mathrm{H} 48$ & 372 \\
\hline 7 & $5 \alpha, 14 \alpha, 17 \alpha$-ergostano $20 \mathrm{~S}$ & $\alpha \alpha \alpha S$ & $\mathrm{C} 28 \mathrm{H} 50$ & 386 \\
\hline 8 & $5 \alpha, 14 \beta, 17 \beta$-ergostano 20R & $\alpha \beta \beta R$ & $\mathrm{C} 28 \mathrm{H} 50$ & 386 \\
\hline 9 & $5 \alpha, 14 \beta, 17 \beta$-ergostano 20S & $\alpha \beta \beta S$ & $\mathrm{C} 28 \mathrm{H} 50$ & 386 \\
\hline 10 & $5 \alpha, 14 \alpha, 17 \alpha$-ergostano $20 \mathrm{R}$ & $\alpha \alpha \alpha \mathrm{R}$ & $\mathrm{C} 28 \mathrm{H} 50$ & 386 \\
\hline 11 & $5 \alpha, 14 \alpha, 17 \alpha$-estigmastano $20 \mathrm{~S}$ & $\alpha \alpha \alpha S$ & $\mathrm{C} 29 \mathrm{H} 52$ & 400 \\
\hline 12 & $5 \alpha, 14 \beta, 17 \beta$-estigmastano 20R & $\alpha \beta \beta R$ & $\mathrm{C} 29 \mathrm{H} 52$ & 400 \\
\hline 13 & $5 \alpha, 14 \beta, 17 \beta$-estigmastano $20 \mathrm{~S}$ & $\alpha \beta \beta S$ & $\mathrm{C} 29 \mathrm{H} 52$ & 400 \\
\hline 14 & $5 \alpha, 14 \alpha, 17 \alpha$-estigmastano $20 \mathrm{R}$ & $\alpha \alpha \alpha \mathrm{R}$ & C29H52 & 400 \\
\hline
\end{tabular}




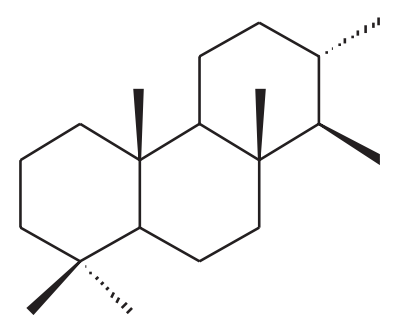

Terpano Tricíclico

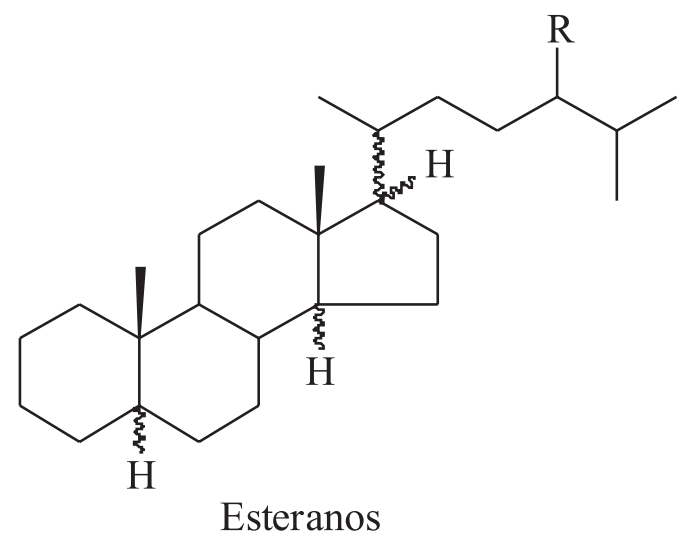<smiles>[X]CC(C)[C@H]1CC[C@H]2[C@@H]3CC[C@H]4[C@@]5(C)CCCC(C)(C)[C@H]5CC[C@]4(C)[C@H]3CC[C@H]12</smiles>

Hopanos Estendidos

Figura 3S. Estruturas de alguns dos biomarcadores citados no texto 